\title{
Naturalizing Husserlian Phenomenology along a Leibnizian Pathway ${ }^{51}$
}

\author{
Jean-Luc Petit \\ Université de Strasbourg \\ jean-luc.petit[]college-de-france.fr \\ Received December 2013; accepted August 2014; published Autumn 2014.
}

\begin{abstract}
A contribution to the history of a formerly hotly discussed, but short-lived scientific project: neurophenomenology ${ }^{52}$, the proposal of weaving together Husserlian phenomenology of consciousness and the neuroscience of brain functioning, this article traces back the opening and closing of an apparent window of opportunity, both in phenomenology and in neuroscience, for the eventually unfulfilled realization of that project.
\end{abstract}

Keywords: neurophenomenology; phenomenology; coherence; autopoiesis; constitution.

Despite a growing literature on naturalizing phenomenology, the subject matter remains controversial. That might be because phenomenology is not intended like natural science to discover a class of facts-such as facts relative to conscious states of human mind. The purpose of phenomenology is rather to reveal the world's mode of appearance to a perceiving subject. In this paper I will put to the test the chances of a recent trend in neuroscience to stand as a satisfactory candidate program for naturalizing a brand of phenomenology at first sight irreducible to natural science: Husserl's transcendantal constitution theory.

1. Mixed perspectives for naturalizing phenomenology.

2. „Konstitution durch Einstimmigkeit der Erfahrung“.

3. Brain orchestra vs single-cell machine-gun .

4. Coherence by way of autopoiesis.

5. In-brain and between-brain coherence.

6. A challenge to pheno-physical reductionism?

\footnotetext{
${ }^{51}$ Acknowledgement to Dr Christopher Macann for the translation.

${ }^{52}$ On the subject of connection between neurophenomenology and enactivism, see note at the end of the article. [eds.]
} 


\section{Mixed perspectives for naturalizing phenomenology}

At the grand congress Actualité cognitive de la Phénoménologie: Les défis de la naturalisation in Bordeaux (19-21 October 1995), the official launch of a cognitive science project for naturalizing Husserlian phenomenology (bridging the gap separating it from natural science), to clarify matters in the discussion I had the happy inspiration of drawing on a transparent a vertical wavy line, on either side of which I put designations of brain processes, and facing each the phenomenological structures of experience I pretended to be "the same thing under another name”. Incidentally, that was the very first expression in a philosophical environment of the hunch-it would assume later the proportions of a scientific hypothesis-that mirror neurons recently identified by Rizzolatti and his team might be the brain substrates of Einfühlung as conceived by Lipps and Husserl. The wavy line was soon retaken on their behalf as code name of the naturalization problem at large by such luminaries as Francisco Varela, Bernard Pachoud, Jean-Michel Roy and Jean Petitot, so that my suggestion was pushed aside and reduced to the triviality of "simply to draw the parallel and leave it unexamined", while they themselves were in the serious business of building the neurophenomenology that the same Varela had heralded (Petitot et al. 1999: 66-67). If you do not mind that I resume a dialogue death has interrupted, I would like to recap my point: $1^{\circ}$ ) systematically correlating constitutive operations in Husserlian phenomenology with functional mechanisms in neuroscience is both meaningful and feasible; $2^{\circ}$ ) far from being a fortuitous analogy, such correlation relies on a fundamental identity of nature: basically it is indeed the same thing; $3^{\circ}$ ) however, lacking a common language to determine that identity, between the subjectivism of phenomenological description and the objectivism of explanation in terms of brain mechanisms the dualism-perhaps only a semantic dualism-is probably insurmountable. But it would be mean of me to move the target after the shot was fired. In this paper I would prefer to examine the chances of another possible way of bridging the gap between transcendantal constitution theory and brain functional systems. That way, without having been followed by Husserl, was at least left open by him as a possibility that his choice of expressions convincingly suggests. And to prove that I am not mean towards Varela I am willing to concede that he spawned the trail in question, if only as a limited application of his ambitious program of neurophenomenology. This time I anchor my proposal in a common language on both sides of the empiricaltranscendental divide. I will capitalize on the fact that instead of two incommensurable discourses, use is made both in phenomenology and neuroscience of a multi-scale concept; I mean a concept applicable to different levels of organization of the living being: coherence. One might speak of coherence at the personal level of the perception and behavior of a subject; but equally at the sub-personal level of assemblies of neurons in the brain. Of course, the question remains how it is possible to move from coherence from the point of view 
of a subject to coherence internal to the brain metabolism: a coherence without a subject, which, however, is not an absolute property of brain anatomical structures, neither might possibly enjoy full autonomy with respect to the material substrate of the brain, but needs to be eventually relativized to functional loops and other rhythms of the organism, as somatic precondition to a coherent subjective experience.

Well-known is the tribute paid to Descartes dualism by transcendantal phenomenology; less so is the growing relevance to Husserl's later oeuvre of Leibniz monadology, a hierarchically organized plurality of monads whose unfolding and refolding are harmoniously synchronized. If Cartesian dualism hinders the circulation of sense between the levels of organization of the living, the ubiquity of sense is the rule in monadological context. In a manuscript from 1935 Husserl warned against localizing mental life in the brain. He relied on an analogy between the organism as a society of cells and society at large. If the intersubjective life of society is not to be tied to the bodies of participants, then the subjective life of a man should not be attached to the society of his bodily cells. However, in an earlier text from 1929 Husserl expressed less negative views while toying with Leibniz' idea of monad as a way of founding subjective life on the system of monadic cells of the organism. Accordingly, Leibniz' favourite metaphor of non-causally interacting synchronized clocks might pave the way to naturalization of phenomenology.

\section{2. „Konstitution durch Einstimmigkeit der Erfahrung"e}

Rejecting every prior ontological assumption, Transcendantal Constitution Theory is uniquely reliant on the intrinsic resources of the perceiving and acting subject of endowing with a sense of being privileged episodes of his experience. Einstimmigkeit der Erfahrung: the tuning of experience with itself proves to be a purely immanent criterion of sense-giving, regardless of the level of organization of experience. A perceiver is prone to intentionally aim at an object in the world as soon as he notices a tuning where there was only discordance: whether between visual field sketches (Abschattungen) and courses of kinaesthesia of the moving organs, between the various sensory modalities, between the body as physical object and the body as lived from within, between the world as viewed by oneself and by others, a tuning he may eventually project at the limit of all possible experience-as in physicsunder the Idea of Nature. Typical of Husserl's transcendental phenomenology is the constant back-reference of the constitutive operations of giving sense of being to the transcendental subject as their bearer. Having sense for... is a relational, not an absolute property. For any entity in experience to be posited as a reality in the world, it needs a special constitutive act of the subject. Having said that, to constitute does not mean to create. The fact that I implement the constitutive operations makes me responsible for the meaningful- 
ness of my experience, but does not turn me into a small creative god. Holding at bay the threat of a solipsistic illusory arbitrariness, the course of our experience harbors a necessity that excludes any caprice even if that necessity is not that of logic and if it manifests itself only in a gradual way. This necessity consists in that even if the discordance is always possible-even if it occurs from time to time-its impact is always limited and does not preclude a more general concordance to recover. Of course, one can always try to assign the privilege of concordance over discordance to the subject, whether the cognitive subject grounding inferences to the future on the basis on prior experience, or the perceiving subject anticipating the constancy of objects in a stable world, or the moral subject who wants to give meaning to his existence and achieves this end in action. Only that we quickly realize that we are caught in a turnstile: on the one hand, each form of concordance in experience should be credited to the constituting subject; on the other hand, the constitutive act of the subject escapes arbitrariness only in relying on the concordance of experience. Is there a way out of that impasse? At first sight it would be like reconciling the materialist monism of neuronal explanation with the dualism of psycho-physical causation. Might concordance possibly emerge from brain metabolism as if summoned to existence by the constitutive power of the transcendental subject, while dispensing with the transcendental stance?

\section{Brain orchestra vs single-cell machine-gun}

Individual cells in the brain are spontaneous oscillators. Specifically the neurons in early visual areas emit a sudden burst of electrophysiological discharge: a potential of action, provided that the preferred stimulus entered their receptive field, the portion of visual field they care for exclusively. A series of potentials of action alternating peaks and troughs gives a wave of electrical activity selectively associated with the stimulus in question and that electrophysiologists see it as a code of the recognition by the perceiving organism of the corresponding aspect of the environment. The propagation of action potentials through synaptic connections from neuron to neuron along the hierarchically organized pathway dedicated to the cognitive treatment of external information obeys the same pattern. It all boils down to a business of anatomical localization and electrical power: a pure matter of energy accumulation somewhere in the brain and energy expenditure for carrying elsewhere the relevant information following a strictly predetermined path of cortical wiring in the white matter of brain tissue. There is no possibility of an action at a distance whether in the brain or between brains, an action, that is to say, an influence without a definite material carrier such as the sensory and motor pathways, an influence from the center to the periphery, from the global to the local, from top to bottom. Such a view of things makes a mystery of performances as unexceptionable as orienting one's attention towards an object 
of interest in the surroundings, conceiving a motor intention and mobilizing the necessary resources to carry out the purpose, or even making oneself understood by the receiver of one's message.

In 1958 Hubel and Wiesel discovered the preferred stimulus of a neuron in the visual cortex of cats and monkeys: they were trying to stimulate a cortical cell by their mentor Stephen Kuffler's method of using slides to project spots onto a screen in front of the animal. Hubel explains:

Then gradually we began to elicit some vague and inconsistent responses by stimulating somewhere in the midperiphery of the retina. We were inserting the glass slide with its black spot into the slot of the ophthalmoscope when suddenly over the audiomonitor the cell went off like a machine gun. After some fussing and fiddling we found out what was happening. The response had nothing to do with the black dot. As the glass slide was inserted its edge was casting a faint but sharp shadow, a straight dark line on a light background. That was what the cell wanted, and it wanted it, moreover, in just one narrow range of orientations. (Hubel \& Wiesel 2004: 661)

A significant change of paradigm took place in neuroscience when the search for single-cell coding of an elementary trait of the environment by a selective burst of electric activity gave way to an investigation of the dynamics of longdistance interactions between brain regions subtending a complex cognitive function. For assemblies of neurons to communicate regardless of their location in the brain, it suffices that their oscillations be transitorily in-phase (phase-locking), a condition requiring no supplementary amount of energy on top of the component neurons activities. Let's consider such in-brain communication by temporal coherence rather than energy consumption or spatial distribution as the nearest possible analogue of Husserl's tuningbased constitution.

\section{Coherence by way of autopoiesis}

On the paradigm shift in neuroscience Varela's ideas have had a profound impact-even if not always acknowledged nowadays. To recap an early article: "Patterns of Life: intertwining Identity and Cognition", dating back to 1997, central to the autonomy of the living being is autopoiesis: a process by which the living being ensures its own production, uniquely characterized by the emergence of a coherence of some kind. The interesting fact about coherence is that while being an effective source of interaction, it is minimally dependent on energy flow, and needs neither controller nor a fixed localization. An autopoietic process, coherence is-so to say-self-produced: it will maintain its organization as long as its basic process resists perturbations and will dissolve when confronted with perturbations that go beyond its viability margins. In the background of such ideas, it is no wonder if Varela's last work applied to emergence of neural assemblies by coherence or synchrony of os- 
cillations, as it is perhaps the simplest, and less objectionable manifestation of autopoiesis in the living being. Let's be more specific about coherence in neuronal networks as an autopoietic mechanism. An individual neuron's action potential is a signal to be understood both ways, as energy expenditure and as information. In visual neurons the information is the noticing of detection of the presence of a preferred stimulus in the cell's receptive field. Might information be decoupled from energy and location? It seems so. A transitorily occurring coherence of the oscillatory activities of distant neuronal groups in the mess of different rhythms in the brain might also convey information about the fact that a new neuronal assembly has just been formed by the grouping of the component neuronal groups through phase coincidence of their respective oscillations. Such information might be relevant to the system in case that neuronal assembly subtended some cognitive function. Yet, the information requires no additional power supply besides that which is consumed by the action potentials of individual neurons. More precisely, energy in the brain is measured by the change in phase amplitude of oscillations: a deflection of electric curve in relation to base-line, a blend of spatial cum intensity dimension of neural activity. In contradistinction, phase synchrony uniquely measures the temporal relationships between neuronal group activities, independently of their amplitude. Two signals cohere with each other if their alternating spikes and troughs succeed synchronously. The constancy of phase similitude (or phase difference) of the two signals during a short lapse of time suggests the existence of a mechanism capable of locking together (phase-locking) at a distance the paces of deployment of both events. The resulting transitorily stable activity pattern over distant brain areas would open up a window of communication between these areas that owes little to the discharge rate of the underlying neurons. Such disassociation between the temporal dimension of brain activity and its dimensions of energy and location opens up a golden avenue for speculations about the possibility of capturing - at a higher level of the description of neural dynamics if not at the finegrained level of individual neurons-the evidence of a purely informational and non-material nor causal interaction between the systems (or subsystems) of the living being. Which not only brings us back to autopoiesis, a mode by which the organism persists in being alive despite the changing energy states in the physical environment, but beyond naturalization of constitution theory by autopoiesis, such move would tend up to legitimize rather uncritically what amounts to an interactionist mind-brain dualism à la Descartes, except that any reference to the thinking subject is discarded in favor of a nonmatter-energy characterization in purely temporal terms of the neuronal basis of cognitive behavior.

In a famous research by Varela and his LENA team at La Salpêtrière (Rodriguez et al, 1999) participants were presented with heavily contrasted photos indistinguishable from random shapes when presented upside down but easi- 
ly recognizable as human faces when presented upright (Mooney figures). Focusing on whole brain $\gamma$ band electric activities $(40 \mathrm{~Hz})$ induced by the stimulus and recorded through electrodes placed on the scalp, they first computed maps of phase amplitude variations of oscillations showing the two expected peaks of spectral power related to presentation of stimulus and to motor response. But they also computed a dynamic mapping of the spatiotemporal distribution of $\gamma$ activities on the scalp from stimulus presentation to motor response, showing that while $\gamma$ activity stayed relatively homogeneous in power emission in all conditions, phase temporal coherence, i.e. phase synchrony differed significantly between electrode pairs. In condition of face perception (but not in no perception condition) there were successively evidenced an increase in phase coherence between parietal and occipital areas coinciding with successful recognition, a sharp desynchronization between all recording sites correlated to the transition from perception to movement, and a final increase in phase coherence between frontal and temporal areas tagging the motor response. From these data the authors inferred the existence of "an integrative mechanism that may bring a widely distributed set of neurons together into a coherent ensemble that underlies a cognitive act”, a mechanism clearly disassociated from power emission in brain tissue but no less endowed with functional significance, since both synchronization and desynchronization of neuronal assembly activities corresponded to behavioral conditions: synchronization to perception and movement, desynchronization to the transition from one cognitive state to the other. They were witnessing, for the first time in human brain research, that the mere temporal profile of a large scale, non-localized brain dynamism might subtend high level cognitive functions. Aren't we tempted to say, pushing to the end the same line of thought: coherence on behavioral level means coherence on the level of neuronal group formation, all else being equal at lower levels (single cells, synapses, molecules, genes, etc.)? It might be risky to interpret it in the sense of a causal autonomy of higher global with respect to underlying local levels, but if we mean descriptive autonomy, that is exactly what the authors argue in their attempt to formulate a theory of emergence accounting for their data: "the relevant variable required to describe these assemblies is not so much the individual activity of the components of the system but the dynamic nature of the links between them". Hence they conclude:

Under this vision, the brain appears as a resourceful complex system that satisfies simultaneously the exogenous and endogenous constraints that arise at each moment by transiently settling in a globally consistent state. These novel views on the brain might throw light on the emergent principles that link neuron and mind, as the large-scale integration of brain activity can be considered as the basis for the unity of mind familiar to us in everyday experience (Varela et al. 2001: 237). 


\section{In-brain and between-brain coherence}

Neuroscience has an object: the brain, not to say an isolated brain. Even brain imagery-a so-called direct view on the brain in act-only describes the brain's functional architecture in terms of statistic averages and baseline subtraction that mask between-subjects differences. Perception and action are still overwhelmingly represented today as internal processes in an isolated brain despite the fact of their retrieval from the flux of interactions of the subject with environment and other subjects. However, if intracerebral synchrony signals interaction (any communication of information) between neural assemblies in a brain, it is tempting to infer by analogy that between-brain synchrony might signal interaction between subjects engaged in communication. Traditional neuroscience used to deal with EEG and fMRI scanner-new social neuroscience deals with dual EEG and Hyperscan. Can we be satisfied to cheer at that progress without considering its uncritically accepted presuppositions? Speaking of communication to describe the interactions between brain regions, we knowingly use a metaphor. No objection to that. This is no longer the case when one inquires into the brain correlates of communication between human subjects engaged in a dialogic relationship. While extending the application of the paradigm of communication by neuronal coherence to the case of ordinary conversation one leaves a clearly metaphorical usage to switch to another mode of expression that deceptively looks like the literal use. The confusion that threatens us is between two very different language games: dialogic and diagnostic. On the one hand, the events in a conversation are narrative episodes of a biography or intentionally aimed at targets of designations and qualifications in the public space for speaking subjects to meet on a common ground and understand each other. On the other hand, one is only dealing with correlative activation foci appearing on the screen of brain imaging scanner. No explanatory gain is provided by the inadvertent commuting of meaning content to and fro: from the domain of conversational interaction between full persons to the domain of neuronal interaction between brain regions, and return. The appearance to the contrary is due to the fact that the tortuous path followed by the explanation makes one lose sight of its illusiveness. In the first place, talking about coherence in terms of communication facilitates the cognitive interpretation of Hebb's law for the formation of neuronal assemblies, which only says, referring to individual cells: "what fires together binds together". Conversely, the principle of effectiveness of synaptic connections is called upon to explain the difference between successful and unsuccessful communication. A move that is not without paying tribute to the vulgar prejudice concerning a necessary involvement of some internal state expressed by the sender of the message and eventually imported in the mind of the receiver, so that to communicate tends to be mistaken as "to communicate a mental state to the partner (Schippers et al. 2010)”. Presumably, the dialogue of lovers enhances the feelings of both-nothing like that 
when filling up a form: regardless of the difference, it does not prevent a successful communication in both conditions. Hebb's law may well account for the effectiveness of the connection by synapses that work rather than those who do not work. Yet projecting the talk of "effectiveness of communication" upon the context of conversation and trying to cope with that effectiveness cannot but create the phantasm of a mental condition specific of successful communications:

if the neural coupling across brains serves as a mechanism by which the speaker and listener converge on the same linguistic act, the extent of coupling between a pair of conversers should predict the success of communication... The findings shown here indicate that during successful communication, speakers' and listeners' brains exhibit joint, temporally coupled, response patterns (Stephens et al. 2010).

Extrapolating towards future technological conditions some speculate on Hyperscan: specifically, two subjects (S1, S2) in their respective scanner will interact by visual signals. S1 sees red or green screen. S1 sends S2 a signal (possibly misleading). S2 wins if he guesses the colour, otherwise S1 has won. Prognosticated result: a global phase coherence of respective brain oscillations including an activity focus in supplementary motor area (SMA) presumably more important in brain of sender than of receiver, etc. Let's part company with such science fiction and pay attention to what the researchers are ultimately aiming at. According to their ingenuous statement, in the future each player will access and influence the other's brain activity: such shortcircuit of any behaviour in the world will reduce society to a correlation "between socially engaged brains" (Montague et al., 2002). Shall the philosopher be accused of bringing an unfair trial against neuroscience if he recalls that society is composed of persons, not of parts of persons, hence not of brains? Even if we do not take account of hazardous speculations of some scientists, it's pretty clear from that example that naturalization of phenomenology guided by the coherence metaphor is engaged in a dead end. But in the very field of neuroscience this window of communication with phenomenology seems to have already closed. Let's see how.

\section{A challenge to pheno-physical reductionism?}

Getting rid of my possibly biased stage-setting, one must concede that the phenomena of brain synchrony and more generally the new path of research on the temporal dimension of brain activity might imply-this is what Varela suggested-a challenge to classical pheno-physical reductionism: the attempt at explaining out consciousness and cognition in terms of brain physicochemistry-but a challenge which is neither unanswerable nor unanswered. In order to measure the full extent of the challenge neuroscientists should not have let go as they did of Varela's autonomy thesis. Certainly, Varela himself 
never claimed a fully-fledged autonomy for the autopoietic emergence of neuronal assemblies, in the sense of an independence from causal influences at lower levels of organization of the material substrate of the brain:

The entire physicochemical constitution is in constant flux; the pattern remains, and only through the organizational invariance can the flux of realizing components be ascertained. In particular, although autopoietic systems are most certainly dissipative chemical systems, a purely matter-energy characterization misses entirely the specific architecture or material circuitry of these very specific chemical systems, which inaugurate the domain of autonomous individuals, and thus of life altogether (Varela, 1997).

However, he no less emphasized the non-locality and the lack of energy demand characterizing the emergence (and disappearance) of synchrony patterns in neuronal networks, a feature that makes these patterns good candidates to be recognized as cerebral correlates of consciousness. That allowed him to support a descriptive (if not causal) autonomy for the high level of description of brain metabolism in relation to the underlying causalities. In that particular he was hardly followed, except perhaps by a few who think they have found the Gestalt laws of organization in the conditions for the emergence of neuronal assemblies (Singer 1999). But the mainstream of researchers thought otherwise. As there is no more absolute synchrony in the brain than absolute simultaneity in a relativistic universe, the emergence of cell assemblies needed to be inserted in a web of entangled functional loops recruiting in different ways all pathways and relay stations of brain activity. (For a synopsis of the cortico-sub-cortical circuits which presumably subtend actions and emotions, see Rolls: The Brain and Emotion 1999). Contextualization in those loops is of a nature to definitely relativize the apparent autonomy of cell assembly dynamics in the cortex at the summit of descending influences from the centre to periphery (as exemplified in attention or in intention). Specifically, the fast rhythms of cell assemblies, presumed neural correlates of cognitive functions, are surrounded - and cannot but be influencedboth by faster rhythms of synapse and cell cycles on one side, and by slower rhythms like circadian, developmental, aging..., on the other. As it is unclear how the phenomenological field of experience-characterized as the retentioprotentional extended window of the "Now" of consciousness-emerges in such polyrhythmic entanglement, we are driven to the conclusion that the gap between physiological mechanisms and experience as it is subjectively lived has not yet been crossed.

In some quarters of recent neuroscience the lure of population (not single cells) coding through holistic activity network patterns seems to give way to a return to, or a refurbishing of Barlow's hierarchical view of brain functioning and to the primacy in that hierarchy of the role of single cell coding: 
Nerve cells are the only means we know about whereby items of information occurring in different part of the brain can be combined; sensory discriminations require the combination of information from different parts of the brain; therefore this operation must be performed by a cell, and if one could record from the cell that did this, one would obtain results at least as good as those of the whole animal. (Barlow 1985: 133-134)

Starting from the assumption that synchrony of activation of individual neurons determines a transitory assembly of neurons in brain (Hebb 1949), but remembering at the same time Barlow's lesson researchers try relating assembly synchrony to a target neuron. In lack of absolute simultaneity in brain circuits the synchrony of source neurons upstream must be relative to a target neuron that integrates the resultant of the sum of their activities downstream. So that solely the neurons discharging in the critical time window of a target neuron (10-30 msec) are synchronous and form a transitory assembly possibly endowed with cognitive significance. A neuron integrating a first assembly might pertain to a second assembly whose neurons activations were synchronous from the viewpoint of another neuron (possibly the member of a third assembly). Adding to that that the same neuron might pertain alternatively or concurrently to different assemblies without change in energy consumption: how such ceaselessly reshuffled groupings might possibly be available to the brain's higher level of representation-not to say to the conscious subject? (Buzsáki 2010)

According to the privileged neuronal coherence hypothesis, synchronization of oscillations as a normal mechanism permits the communication between neurons or neuron assemblies subtending perception and motor behaviour. Desynchronization of oscillations is also a normal process of decoupling neuron assemblies so as to turn possible the transition from one cognitive state or behaviour to another in experience. But synchronization and desynchronization need not be always functional. Associate to pathologic symptoms of neuromuscular diseases (tremor in Parkinson, Tourette syndrome, dystonia, etc.) is an abnormal unselective pattern of synchronization between motor system areas. A review of works on essential tremor in Parkinson disease shows impressive average maps of long distance non selective synchronization between controlateral primary motor cortex where oscillations are recorded at double the frequency of muscular tremor, and various areas whose oscillations are coherent with that motor cortex, including lateral and medial premotor cortices, somatosensory cortex, thalamus, basal ganglia and ipsilateral cerebellum (Schnitzler \& Gross 2005). Such perturbation proves brain selective synchronization to depend on a subtle balance between excitatory and inhibitory pathways in the circuitry connecting basal ganglia to thalamus to cortex. Reciprocally, dementia in Alzheimer disease has been linked to a reduced synchronization of high frequency oscillations. The ideas of emergence and phase locking backing the coherence hypothesis with its neighbouring 
suggestion of the possibility of stabilization of cognitively functional activity patterns in the whole brain dynamics prepare us insufficiently to deal with dysfunctions.

Not to extend the list, we'll just add that a rebuff to the non-localization tendency of coherency theories is perceptible in the search for a controlling centre in the brain responsible for modulating or even inducing the rhythms recorded at the higher level of cortical neuronal assemblies, as if the need for explanation could not satisfy itself with the sole description of emerging spatiotemporal patterns of activity. In this regard, we cannot ignore the contributions of those who try answering the question of the sources of brain rhythms and eventually point at thalamus as a possible multi-purpose pace-maker for oscillatory activities anywhere in cortical areas. For example, subjects bearers of electrodes both on the scalp and in thalamus are submitted to a semantic memory retrieval task such as emitting a word in response to the presentation of a couple of related or unrelated words ("desert" + "hump" => "camel"). The result is that during semantic recall a drop in low-frequency rhythms power at 1-2s post-stimulus followed by an increase at 2-3s in fast rhythm power registered at thalamic and cortical electrodes. Advancing their theoretical model of thalamus as pacemaker for cortical rhythms, the authors emphasize the fact that here again all is a question of balance between excitatory and inhibitory influences from underneath, not of emerging stable patterns. Lowfrequency rhythms controlled by inhibitory projections from thalamic reticular formation to thalamo-cortical cells counterbalance high-frequency rhythms controlled by excitatory cortico-thalamo-cortical pathway (Slotnick et al. 2002).

\section{Conclusion}

-Has phenomenology been satisfactorily naturalized in a neuroscience? It might well have been the case if the autopoietic interpretation of emergent network coherence in cortex had prevailed. But in a multi-scale neuroscience the intra-brain and inter-brain distant interactions at the basis of cognitive states enjoy no privilege in relation to the cascading levels of organization of the living organism, from molecules to behaviour to phylogenesis. The possible window of dialogue Varela opened up-if our interpretation is correctbetween the neurophysiology of neuronal coherence and a constitutive phenomenology based on Einstimmigkeit der Erfahrung seem to have had a short life time indeed, since the current orientation of the research community rather favors embedding the time structure of transitory occurring neuronal assemblies in the larger context of brain rhythms at the multiple levels of organization of the living being. So, if one wanted an explanation in mechanistic terms of our capability of giving sense of being to salient episodes of experience provided they kept a seamless tuning, that question would be broken up 
in so many subquestions that their addition would leave one with a sense of indefinite postponement. A sobering experience, considering that the temporal structure of brain metabolism in the image that Varela displayed was of a kind to lure some phenomenologists in the belief of having reached a satisfactory response.

Note: Some would prefer to know more about the connection between neurophenomenology and enactivism, a concept that I did not use in this paper. To recap, neurophenomenology is a scientific program aimed at integrating the first person description of subjective experience and the third person explaining of cognition through brain dynamics. Enactivism, in cognitive science, is a stance alternative to representationalism and to dualism, conceives cognition as contingent upon sensori-motor interaction between an organism and its environment. A link between neurophenomenology and enactivism was provided to Varela and his followers by the phenomenology of the lived body developed by Merleau-Ponty in the wake of the later part of Husserl's philosophy.

\section{Bibliography}

Barlow, H.B. 1985. The role of single neurons in the psychology of perception. The Quarterly Journal of Experimental Psychology, 37A: 121-145.

Buzsáki, G. 2010. Neural Syntax: Cell Assemblies, Synapsembles, and Readers. Neuron, 68: 362-385.

Hebb, D.O. 1949. The Organization of Behavior: A Neuropsychological Theory. Wiley, New York.

Hubel, D.H. Wiesel, T.N., 2004. Brain and Visual Perception. The story of a twenty-fiveyear collaboration. Oxford University Press, Oxford.

Husserl, E. 1929, ms C8II.

Husserl, E. 1935, ms BI16.

Montague, P.R., Berns, G.S., Cohen, J.D., McClure, S.M., Pagnoni, G., Dhamala, M., Wiest, M.C., Karpov, I., King, R.D., Apple, N., Fisher, R.E. 2002. Hyperscanning: Simultaneous fMRI during linked social interactions. NeuroImage, 16: 1159-1164.

Petitot, J., Varela, F.J., Pachoud, B., Roy, J-M. 1999. Naturalizing Phenomenology. Issues in contemporary Phenomenology and Cognitive Science. Stanford University Press, Stanford, California.

Rodriguez, E., George, N., Lachaux, J-Ph., Martinerie, J., Renault, B., Varela, F.J. 1999. Perception's shadow: long-distance synchronization of human brain activity. Nature, 397: 430-433.

Rolls, E. 1999. The Brain and Emotion. Oxford University Press, Oxford. 
Schippers, M.B., Roebroeck, A., Renken, R., Nanetti, L., Keysers, Ch. 2010. Mapping the information flow from one brain to another during gestural communication. PNAS, 107, 20: 9388-9393.

Schnitzler, A., Gross, J. 2005. Normal and pathological oscillatory communication in the brain. Nature Reviews Neuroscience, 6: 285-296.

Singer, W. 1999. Neuronal Synchrony: A versatile code for the definition of relations? Neuron, 24: 49-65.

Slotnick, S.D., Moo, L., Kraut, M.A., Lesser, R.P., Hart Jr, J. 2002. Interactions between thalamic and cortical rhythms during semantic memory recall in human. PNAS, 99, 9: 6440-6443.

Stephens, G.J., Silbert, L.J., Hasson, U. 2010, Speaker-listener neural coupling underlies successful communication, PNAS, 107, 32: 14425-14430.

Varela, F.J. 1997. Patterns of Life: Intertwining Identity and Cognition, Brain and Cognition 34: 72-87.

Varela, F.J., Lachaux, J-Ph., Rodriguez, E., Martinerie, J. 2001. The Brainweb: Phase synchronization and large-scale integration. Nature Reviews Neuroscience, 2: 220-239. 